\title{
POTENTIAL HAZARDS FROM NEUTRINO RADIATION AT MUON COLLIDERS
}

\author{
Bruce J. King, Brookhaven National Laboratory ${ }^{1}$
}

\begin{abstract}
High energy muon colliders, such as the TeV-scale conceptual designs now being considered, are found to produce enough high energy neutrinos to constitute a potentially serious off-site radiation hazard in the neighbourhood of the accelerator site. A general characterization of this radiation hazard is given, followed by an order-of-magnitude calculation for the off-site annual radiation dose and a discussion of accelerator design and site selection strategies to minimize the radiation hazard.
\end{abstract}

\section{INTRODUCTION}

Current conceptual designs for muon colliders [1] envisage large currents of opposing positively and negatively charged muons circulating in a collider ring until decay into neutrinos and electrons:

$$
\begin{aligned}
& \mu^{-} \rightarrow \nu_{\mu}+\overline{\nu_{\mathrm{e}}}+\mathrm{e}^{-}, \\
& \mu^{+} \rightarrow \overline{\nu_{\mu}}+\nu_{\mathrm{e}}+\mathrm{e}^{+} .
\end{aligned}
$$

This will produce an intense disk of neutrinos emanating out in the plane of the collider ring. The vertical angular divergence of the neutrino disk can be characterized by the spread in the relative angle between the neutrino and muon directions and, from relativistic kinematics, the neutrinos in the forward hemisphere in the muon rest frame are boosted, in the laboratory frame, into a narrow cone with an opening half-angle,

$$
\theta_{\nu} \simeq \sin \theta_{\nu}=1 / \gamma=\frac{m_{\mu} c^{2}}{E_{\mu}} \simeq \frac{10^{-4}}{E_{\mu}[\mathrm{TeV}]}
$$

with $\gamma$ the relativistic boost factor of the muon, $E_{\mu}$ the muon energy and $m_{\mu}$ the muon rest mass.

The vertical angular spread of the neutrino disk could, in principle, also receive contributions from the angular spread of the muon beam. However, for reasonable magnet lattice designs this will usually produce negligible additional divergence everywhere around the collider ring except at the final focus regions around collider experiments.

The potential radiation hazard comes from the showers of ionizing particles produced in interactions of neutrinos in the soil and other objects bathed by the disk. The tiny interaction cross-section for neutrinos is greatly compensated by the huge number of high energy neutrinos produced at muon colliders.

\footnotetext{
1 web page: http://pubweb.bnl.gov/people/bking/ email: bking@bnl.gov. This work was performed under the auspices of the U.S. Department of Energy under contract no. DE-AC02-98CH10886.
}

\section{QUANTITATIVE DERIVATION OF RADIATION DOSE}

A quantitative expression for the radiation dose received by a person from the decay of $N_{\mu}$ muons of each sign, at tangential distance $L$ from an idealized perfectly circular muon collider ring and in the plane of the ring is given by:

$$
\begin{gathered}
D^{\text {ave }}=\frac{N_{\mu}}{L^{2}} \int_{0}^{2 \pi} \frac{d \theta}{2 \pi} \cdot \frac{1}{4 \pi} \frac{d \Omega^{\prime}}{d \Omega}(\theta) \times \\
\sum_{\text {Ltype }, i=1,4} \int_{0}^{1} d x f^{i}(x) \sigma^{i}\left(E_{\nu}\right) d^{i}\left(E_{\nu}\right),
\end{gathered}
$$

where $\mathrm{E}_{\nu}$ is a function of both integration variables, $x$ and $\theta$ and the variables and form of the expression are explained in the following paragraphs.

In principle, neutrinos can be emitted in all directions relative to the muon trajectory at decay so the angle between the muon beam and the neutrino direction, $\theta$, is formally integrated over all muon directions around ring, However, equation 11 shows that most of the contribution to the radiation dose will come from neutrinos oriented within of order $1 / \gamma$ or less from the muon beam direction, so the size of the collider ring can be ignored. Clearly, a fraction $\frac{d \theta}{2 \pi}$ of the muons will decay in the angular increment $d \theta$ and, for the reasonable assumption that the muon beams are unpolarized on average, the neutrino decays will be isotropic in muon rest frame and the fraction of neutrino decays per unit solid angle in the laboratory rest frame is is $\frac{1}{4 \pi} \frac{d \Omega^{\prime}}{d \Omega}(\theta)$, where primed coordinates denote the muon rest frame and unprimed coordinates the laboratory rest frame. Relativistic kinematics gives:

$$
\frac{d \Omega^{\prime}}{d \Omega}=\gamma\left(1+\beta \cos \theta^{\prime}\right) \frac{d \theta^{\prime}}{d \theta},
$$

$\gamma=9.46 \times 10^{3} \times E_{\mu}[\mathrm{TeV}]$ and $\beta \simeq 1$ for realistic collider energies. This allows the integration over the laboratory angle, $\theta$ in equation 3 to be replaced by an integration over the angle in the muon rest frame, $\theta^{\prime}$.

A biological target in the radiation disk is tangent to the collider ring in two places and so will receive neutrinos from the decays of both positive and negative muons. Therefore, the neutrino type index, i, runs over all 4 neutrino types $-\overline{\nu_{\mathrm{e}}}, \nu_{\mu}, \overline{\nu_{\mu}}$ and $\nu_{\mathrm{e}}$.

The energy probability distribution in the muon rest frame for the production of neutrino type i is $d x \cdot f_{x}^{i}$, with $x$ defined as the fraction of the maximum neutrino energy in the muon rest frame: $E_{\nu}^{\prime}=\frac{x \cdot m_{\mu} c^{2}}{2}$. The explicit form for $f$ is known to be: $f=6 \cdot x^{2}-4 . x^{3}$ for unpolarized muontype neutrinos or anti-neutrinos and $f=12 . x^{2}-12 . x^{3}$ 
for unpolarized electron-type neutrinos or anti-neutrinos. Boosting to the laboratory frame gives

$$
E_{\nu}\left(x, \cos \theta^{\prime}\right)=x \cdot \frac{E_{\mu}}{2}\left(1+\beta \cos \theta^{\prime}\right) .
$$

The cross-section per nucleon, $\sigma^{i}\left(E_{\nu}\right)$, is expressed, for now, in the same units of length as $\mathrm{L}$ and $d^{i}\left(E_{\nu}\right)$ is the average radiation dose from a neutrino of type $\mathrm{i}$ and energy $E_{\nu}$ interacting in each nucleon of a biological target, assuming the equilibrium approximation and expressed in the same units as $D^{\text {ave }}$.

Most of the ionization energy dose deposited in a person will come from interactions in the soil and other objects in the person's vicinity rather than from the more direct process of neutrinos interacting inside a person. At $\mathrm{TeV}$ energy scales, much less than one percent of the energy flux from the daughters of such interactions will be absorbed in the relatively small amount of matter contained in a person, with the rest passing beyond the person.

Equation 3 implicitly assumes the simplifying "equilibrium approximation" that the ionization energy absorbed by a person is equal to the energy of the showers initiated by interactions in that person.

It seems reasonable to assume that the equilibrium approximation should give either a reasonable estimate or a conservative overestimate of the radiation dose absorbed by a person for most of the innumerable possible distributions of matter. From conservation of energy, it would clearly be a good approximation for the reference case of a homogeneous medium of any density sufficient that the radial extent of the hadronic and electromagnetic showers initiated by neutrino interactions is small compared to the height of the neutrino radiation disk. In realistic geometries, some of the shower energy will typically leak out to beyond the extent of the neutrino disk through low density regions of air etc., presumably decreasing the radiation dose to below the equilibrium estimate.

The radiation dose in units of Sieverts (Sv) is numerically almost equal to the energy deposition in a biological target in units of $\mathrm{J} / \mathrm{kg}$ for the energetic hadronic and electromagnetic showers from neutrino interactions. Therefore, if $k^{i}$ is defined to be the weighting factor for converting from neutrino energy to radiation dose for a neutrino of type $\mathrm{i}$ then $k^{i}$ will be numerically equal to the average fraction of the interaction energy ending up as electrons or hadrons.

The radiation dose per neutrino of energy $E_{\nu}$ is then given by $d^{i}\left(E_{\nu}\right)[S v] \simeq 1.6 \times 10^{-7} \times k^{i} \times E_{\nu}[T e V] \times\left(10^{3} \times\right.$ $\left.N_{\text {Avogadro }}\right)$, where $1.6 \times 10^{-7}$ is the conversion factor from Joules to $\mathrm{TeV}$ and the numerical factor $10^{3} \times N_{\text {Avogadro }}$ arises because Sieverts are defined as energy absorbed per kilogram rather than per nucleon.

Because neutrino cross-sections are almost linear with energy in the $\mathrm{TeV}$ range they can be expressed as $\sigma^{i}\left(E_{\nu}\right)\left[\mathrm{cm}^{2}\right] \simeq \sigma_{R}^{i}\left[\mathrm{~cm}^{2} / T e V\right] \times E_{\nu}[\mathrm{TeV}]$, where the "reduced cross section", $\sigma_{R}^{i}$, can be approximated as a constant over a fairly large energy range.
On making these substitutions and integrating over the muon angle, equation 3 can be rewritten as:

$$
\begin{aligned}
& D^{a v e}[S v]=4.5 \times 10^{15} \times \frac{N_{\mu} \times\left(E_{\mu}[\mathrm{TeV}]\right)^{3}}{(L[k m])^{2}} \times \\
& \sum_{\nu \text { type }, i=1,4} \sigma_{R}^{i}\left[\mathrm{~cm}^{2} / \mathrm{TeV}\right] \times k^{i} \times \int_{0}^{1} d x f^{i}(x) \cdot x^{2} \text {, }
\end{aligned}
$$

where the units of all dimensioned variables are given in square brackets.

The dominant interaction processes of TeV-scale neutrinos are charged current (CC) and neutral current (NC) deep inelastic scattering off nucleons:

$$
\begin{aligned}
& \nu+\text { nucleon } \rightarrow \mu(\text { or e })+\text { hadrons }(\mathrm{CC}) \\
& \nu+\text { nucleon } \rightarrow \quad \nu+\text { hadrons }(\mathrm{NC})
\end{aligned}
$$

It is the subsequent interactions of the daughter hadrons and electrons, initiating showers of ionizing particles, that constitute the bulk of the radiation hazard. In contrast, that part of the neutrino energy transferred into daughter neutrinos or muons will almost all be transported to outside the radiation disk rather than contributing to the energy absorbtion of a person within the disk.

The numerical calculations for equation 6 are summarized in table 1 . Substituting this into equation 6 gives:

$$
D^{a v e}[S v]=3.7 \times 10^{-23} \times \frac{N_{\mu} \times\left(E_{\mu}[T e V]\right)^{3}}{(L[k m])^{2}} .
$$

Table 1: Contributions to the radiation dose from the different types of neutrino interactions. The reduced crosssecttion, $\sigma_{R}^{i}$, is specified for $100 \mathrm{GeV}$ neutrinos and using a simple model for the nucleon in which the crosssection ratio for neutrinos to anti-neutrinos was assumed to be $2: 1$ and ignoring the small differences between the average hadronic fractions for $\mathrm{NC}$ and $\mathrm{CC}$ interactions. The reduced cross-section and product are in units of $10^{-35} \mathrm{~cm}^{2} / \mathrm{TeV}$.

\begin{tabular}{|ccccc|}
\hline int., $i$ & $\sigma_{R}^{i}$ & $k^{i}$ & $\left\langle x^{2}\right\rangle^{i}$ & $\sigma_{R}^{i} \cdot k^{i} \cdot\left\langle x^{2}\right\rangle^{i}$ \\
\hline$\nu_{\mu}-C C$ & 0.722 & 0.458 & 0.533 & 0.176 \\
$\nu_{\mu}-N C$ & 0.226 & 0.458 & 0.533 & 0.055 \\
$\nu_{\mathrm{e}}-C C$ & 0.722 & 1.000 & 0.400 & 0.289 \\
$\nu_{\mathrm{e}}-N C$ & 0.226 & 0.458 & 0.400 & 0.041 \\
$\overline{\nu_{\mu}}-C C$ & 0.375 & 0.292 & 0.533 & 0.058 \\
$\overline{\nu_{\mu}}-N C$ & 0.131 & 0.292 & 0.533 & 0.020 \\
$\overline{\nu_{\mathrm{e}}}-C C$ & 0.375 & 1.000 & 0.400 & 0.150 \\
$\overline{\nu_{\mathrm{e}}}-N C$ & 0.131 & 0.292 & 0.400 & 0.015 \\
\hline SUM & & & & 0.804 \\
\hline
\end{tabular}

It will now be shown that the radiation intensity would be expected to vary greatly around the neutrino disk, depending on the detailed design of the collider ring magnet lattice, so the value of $D_{\text {ave }}$ by itself is not sufficient to assess the radiation hazard for any particular collider design. For example, it is clear from the derivation of equation 7 
that the radiation contribution tangent to the collider ring at a dipole bending magnet will be proportional to the beam's bending radius at the magnet, which is inversely proportional to the magnetic field strength.

For even bigger variations, tangents to the collider ring at anywhere other than a dipole magnet the muon currents will travel in straight line trajectories and the neutrinos will line up as local radiation "hot spots" in the radiation disk cones of more intense radiation with characteristic opening half-angles of $\theta_{\nu}=1 / \gamma$.

The contribution from straight sections is given by an equation analagous to equation 3 :

$$
\begin{array}{r}
D^{s s}=\frac{f^{s s} \times N_{\mu}}{L^{2}} \times \frac{\gamma^{2}}{\pi} \times \\
\sum_{\text {Ltype }, i=1,2} \int_{0}^{1} d x f^{i}(x) \sigma^{i}\left(E_{\nu}\right) d^{i}\left(E_{\nu}\right),
\end{array}
$$

where $f^{s s}$ is the length of the straight section as a fraction of the collider circumference

$$
f^{s s}=L / C
$$

and the factor $\gamma^{2} / \pi$ is the fraction of neutrinos decaying in the forward direction per unit solid angle after being boosted from isotropic decays in the muon rest frame into the laboratory frame.

The summation in equation 8 is now only over the 2 neutrino types produced by the sign of muon travelling in the considered direction, i.e., either $\overline{\nu_{\mathrm{e}}}$ and $\nu_{\mu}$ for $\mu^{-}$decays or $\overline{\nu_{\mu}}$ and $\nu_{\mathrm{e}}$ for $\mu^{+}$decays (equation 11 ). The summed contributions in table 1 for $\mu^{+}$and $\mu^{-}$are very nearly equal, so it is reasonable to use the average contribution, $0.402 \times 10^{-35} \mathrm{~cm}^{2} / \mathrm{TeV}$, for either sign.

Following a similar derivation to that for equation 7 the numerical value for the dose is:

$$
D^{s s}[S v]=1.1 \times 10^{-18} \times \frac{f^{s s} \times N_{\mu} \times\left(E_{\mu}[T e V]\right)^{4}}{(L[k m])^{2}} \text {. }
$$

The radiation cones from the final focus regions around collider experiments are important exceptions to equation 10, since the muon beam itself will have an angular divergence in these regions that may be large enough to spread out the neutrino beam by at least an order of magnitude in both $\mathrm{x}$ and $\mathrm{y}$.

More detailed calculations to check and refine these calculations, using Monte Carlo-based particle tracking computer simulations, are in progress.

On comparing equations 7 and 10 it is easily seen that the length of straight section to produce an extra contribution equal to the planar average dose, $l_{\text {equiv }}$, is approximately:

$$
l_{\text {equiv }}[\text { meters }] \simeq 0.034 \times \frac{C[k m]}{E_{\mu}[T e V]} \simeq \frac{0.71}{B_{\text {ave }}[T]}
$$

where the final expression uses the relation between muon energy, ring circumference and and average bending magnetic field in units of Tesla:

$$
C[k m]=\frac{2 \pi \cdot E_{\mu}[T e V]}{0.3 \cdot B_{a v e}[T]}
$$

valid for a circular ring.

Two mitigating factors come into play at many- $\mathrm{TeV}$ energies to reduce the radiation rise with energy:

1. the neutrino cross section begins to rise significantly less rapidly than linearly with neutrino energy

2. the radiation disk (or cone) becomes so narrow that the "equilibrium approximation" is no longer accurate because much of the induced shower of charged particles leaks out transversely beyond the extent of the radiation disk. The "cut-off" width at which the equilibrium approximation will begin to fail badly is the typical transverse shower size in whatever medium is initiating the showers. This will be of order a meter for typical solids. (As an aside, it will be hundreds of meters in air so the equilibrium approximation will be overly conservative at all collider energies for people in open areas rather than surrounded by massive objects.)

\section{STRATEGIES TO MINIMIZE OFF-SITE RADIATION DOSES}

Because of the strong dependence on muon energy, the radiation levels rapidly become a serious design constraint for colliders at the $\mathrm{TeV}$ scale and above. For illustration, table 2 gives the predicted radiation levels for some example muon collider parameter sets [3]. For comparison, the U.S. federal off-site radiation limit is $10^{-3} \mathrm{~Sv} /$ year, which is of the same order of magnitude as the typical background radiation from natural causes (i.e. 0.4 to $4 \times 10^{-3} \mathrm{~Sv} / \mathrm{yr}$ [2]) and it is assumed that acceptable radiation levels must be considerably lower than these values.

As a desirable design strategy for all energies, it is clear that great care must be taken to minimize or eliminate long straight sections in the collider ring. For example, the magnet lattice could consist partly or entirely of dual function magnets, where the beam focusing and bend are accomplished in the same magnets. Optionally, it might be convenient to retain one or two long straight sections by constructing radiation enclosures around where their radiation hot spots exit the ground.

Perhaps the most direct way of decreasing the radiation levels is to greatly decrease the muon current. This can be done either by sacrificing luminosity (as in the $4 \mathrm{TeV}$ parameter set of table 2) or, more attractively, by increasing the luminosity per given current through better muon cooling or other technological advances.

Further, one might consider placing the accelerator deep underground so the radiation disk won't reach the surface for some distance. For the example of a very flat region of the Earth the exit distance to the surface $\mathrm{L}_{\text {exit }}$ will be related to the collider depth, $D$, and the Earth's radius, $R_{E}=6.4 \times 10^{6} \mathrm{~m}$, by $L_{\text {exit }}=\left(2 \times D \times R_{E}\right)^{1 / 2}$, where the three parameters are in consistent units of length, e.g., meters. Substituting into equations 7 and 10 gives 


$$
D_{\text {exit }}^{\text {ave }}[S v]=2.9 \times 10^{-24} \times \frac{N_{\mu} \times\left(E_{\mu}[\mathrm{TeV}]\right)^{3}}{(D[\mathrm{~m}])}
$$

and

$D_{\text {exit }}^{s s}[S v]=4.1 \times 10^{-24} \times \frac{l[m] \times B_{a v e} \times N_{\mu} \times\left(E_{\mu}[T e V]\right)^{3}}{(D[m])}$

respectively, where equations 11 and 12 have been substituted into the second of these equations.

It is seen that the radiation dose at exit falls inversely with collider depth. The quadratic dependence of the depth on $L_{\text {exit }}$ means that exit distances of order $10 \mathrm{~km}$ are easily achievable, but achieving an $L_{\text {exit }}$ of order $100 \mathrm{~km}$ is already starting to require a prohibitively large depth.

Further speculative options that have been discussed include (i) tilting the ring to take best advantage of the local topography, (ii) placing the collider ring on a hill so the radiation disk passes harmlessly above the surroundings and, even more speculatively, (iii) spreading out and diluting the neutrino radiation disk by continuously sweeping the muon beam orbit in a vertical plane using dipole corrector magnets.

Even when the preceding strategies have been used, the strong rise in neutrino energy probably dictates that muon colliders at $\mathrm{CoM}$ energies of beyond a few $\mathrm{TeV}$ will probably have to be constructed at isolated sites where the public would not be exposed to the neutrino radiation disk at all. This would definitely be required for the $10 \mathrm{TeV}$ and 100 $\mathrm{TeV}$ parameter sets of table 2. Because of the additional costs this would involve, these will presumably be "second generation" machines, arriving after the technology of muon colliders has been established in one or more smaller and less expensive machines built at existing HEP laboratories.

In conclusion, some order-of-magnitude calculations have been presented which show that the neutrino-induced radiation hazard might be a very serious problem for high energy muon colliders. The neutrino radiation problem appears to impose severe constraints on the site selection for a muon collider complex and on the layout of the complex.

It is speculated that the highest energy muon (and hadron) colliders and their associated neutrino radiation disks may be required to be enclosed within a huge new world HEP laboratory somewhere where there is a large area of cheap, sparsely populated land.

\section{ACKNOWLEDGEMENTS}

This paper has benefitted greatly from discussions with collaborators in the muon collider collaboration, particularly Dr. Robert Palmer and Dr. Nikolai Mokhov.

\section{REFERENCES}

[1] The Muon Collider Collaboration, "Status of Muon Collider Research and Development and Future Plans", to be submitted to Phys. Rev. E.
[2] The Particle Data Group, Review of Particle Physics, Phys. Rev. D54 (1996).

[3] B.J. King, "Discussion on Muon Collider Parameters at Center of Mass Energies from $0.1 \mathrm{TeV}$ to 100 TeV", 19 June, 1998, Submitted to Proc. Sixth European Particle Accelerator Conference (EPAC'98), Stockholm, Sweden, 22-26 June, 1998. Available at http://pubweb.bnl.gov/people/bking. 
Table 2: Radiation dose estimates for the example muon collider parameter sets of reference [3].

\begin{tabular}{|r|ccccc|}
\hline $\begin{array}{c}\text { center of mass energy, } \mathrm{E}_{\mathrm{CoM}} \\
\text { description } \\
\text { luminosity, } \mathcal{L}\left[\mathrm{cm}^{-2} \cdot \mathrm{s}^{-1}\right]\end{array}$ & $\begin{array}{c}0.1 \mathrm{TeV} \\
\mathrm{MCC} \text { para. set } \\
1.2 \times 10^{32}\end{array}$ & $\begin{array}{c}1 \mathrm{TeV} \\
\mathrm{LHC} \text { complement } \\
1.0 \times 10^{34}\end{array}$ & $\begin{array}{c}4 \mathrm{TeV} \\
\mathrm{E} \text { frontier } \\
6.2 \times 10^{33}\end{array}$ & $\begin{array}{c}10 \mathrm{TeV} \\
2^{\text {nd }} \text { gen. } \\
1.0 \times 10^{36}\end{array}$ & $\begin{array}{c}100 \mathrm{TeV} \\
\text { ult. E scale } \\
4.0 \times 10^{36}\end{array}$ \\
\hline \hline relevant collider parameters: & & & & & \\
circumference, $\mathrm{C}[\mathrm{km}]$ & 0.3 & 2.0 & 7.0 & 15 & 100 \\
ave. bending B field $[\mathrm{T}]$ & 3.5 & 5.2 & 6.0 & 7.0 & 10.5 \\
$\left(\mu^{-}\right.$or) $\mu^{+} /$bunch, $\mathrm{N}_{0}\left[10^{12}\right]$ & 4.0 & 3.5 & 3.1 & 2.4 & 0.18 \\
ave. current $[\mathrm{mA}]$ & 15 & 15 & 0.67 & 15 & 60 \\
beam power $[\mathrm{MW}]$ & 1.0 & 10 & 0.46 & 24 & 4.2 \\
$\left(\mu^{-}\right.$or) $\mu^{+}$bunch rep. rate, $\mathrm{f}_{\mathrm{b}}[\mathrm{Hz}]$ & 8.4 & 1.3 & 58 & 170 \\
time to beam dump, $\mathrm{t}_{\mathrm{D}}\left[\gamma \tau_{\mu}\right]$ & no dump & 0.5 & 0.5 & no dump & 0.5 \\
effective turns/bunch & 519 & 493 & 563 & 1039 & 985 \\
\hline \hline neutrino radiation parameters: & & & & & \\
collider reference depth, $\mathrm{D}[\mathrm{m}]$ & 10 & 125 & 300 & 300 & 300 \\
$\nu$ beam distance to surface $[\mathrm{km}]$ & 11 & 40 & 62 & 62 & 62 \\
$\nu$ beam radius at surface $[\mathrm{m}]$ & 24 & 8.4 & 3.3 & 1.3 & 0.13 \\
str. sect. length for 10x ave. rad., $\mathrm{L}_{\mathrm{x} 10}[\mathrm{~m}]$ & 1.9 & 1.3 & 1.1 & 1.0 & 2.4 \\
ave. rad. dose in plane $[\mathrm{mSv} / \mathrm{yr}]$ & $3 \times 10^{-5}$ & $9 \times 10^{-4}$ & $9 \times 10^{-4}$ & 0.66 & 6.7 \\
\hline
\end{tabular}

